\title{
Experimental Study on Characteristics of Short Wave Irregularity in Urban Rail Transit
}

\author{
Jian FANG, Wenwen ZHENG and Xiaoyan LEI ${ }^{1}$ \\ Engineering Research Center of Railway Environment Vibration and Noise of the \\ Ministry of Education, East China Jiaotong University, Nanchang, Jiangxi Province \\ 330013, China
}

\begin{abstract}
Based on the measured irregularity data of Shanghai rail transit 11\# line, firstly from the angle of time domain the amplitude stability and amplitude distribution characteristics of the rail surface short wave irregularity whose wavelengths are in the range of $0.01 \sim 1 \mathrm{~m}$ are analyzed. Secondly from the point of view of frequency domain by using the maximum entropy spectrum method the short wave power spectral density is estimated. Then the power spectral density curves are compared with the short spectrum of Wang Lan and Sato and the different track structure effects on the states of the rail surface irregularities are also analyzed. The research show that the amplitude distribution of track surface short wave irregularity is close to the normal distribution. And the maximum amplitudes of some sections in Jiading Xincheng - Malu and Nanxiang - Taopu saemaul undong exceed $2.0 \mathrm{~mm}$. In the section of Liziyuan-Shanghai West Railway Station, the power spectral density curves of track structure with the supporting block, DTIII-2 type fasteners and with the conventional integral track bed are also similar to that of Sato spectrum. And it is obviously superior to that of other track structures in this section.
\end{abstract}

Keywords. Rail transit, short wave irregularity, amplitude distribution, power spectral density, track structure

\section{Introduction}

In recent years the development momentum of Chinese railways has become more and more rapid, especially in the area of urban rail transit construction. However, serious vibration and noise problems caused by the urban rail transit generally become more and more obvious. The root cause is high frequency vibration caused by short wave irregularity on rail surface. Generally the wavelength of rail surface short wave irregularity ranges from several centimeters to dozens of centimeters and its amplitude is less than $2 \mathrm{~mm}[1,2]$. It is the main excitation source of vehicle-track system vibration, which will cause high-frequency impact force [3-5] and damage the track structure. The high-frequency impact force even causes rail and axle breakage and malignant derailment [6], which endangers driving safety [7]. Therefore, the 
management of rail surface short wave irregularity is particularly important in rail maintenance and repair. The basis for evaluating rail surface short wave irregularity in overseas rail transit is mainly the level spectrum standard of rail surface short wave irregularity developed by International Organization for Standardization (ISO) for studying wheel-rail noise [8]. Under this standard, the rail roughness level of the Netherlands and Sweden railways is tracked and measured by Hiensch [9] and Verheijen [10]. Based on this, the rail surface short wave roughness level is evaluated and mathematically predicted by Nielsen [11] At present, due to the restriction of rail short wave irregularity measure and analysis methods, short wave irregularity of urban rail transit has not been fully studied in China. Especially, there are many urban rail lines in our country, and there are differences between maintenance period and curing methods of each line. The distribution characteristics and development rules of short wave irregularity on rail surface are also different. Therefore, it is of great significance to study and analyze the rail surface short wave irregularity.

This paper is based on the actual measurement of Shanghai rail transit Line 11\#. Firstly, the amplitude distribution law of measured data in time-domain is counted, and the power spectrum density curve is compared and analyzed in frequency domain, which can provide reference for effective vibration and noise reduction measures and treatment schemes for urban rail transit.

\section{Profile of Field Test}

In this paper, the CAT rail corrugation measuring instrument manufactured by Rail Measurement Company in UK is used to collect data from the uplink and downlink lines of Shanghai rail transit Line 11\#. The sampling interval is 500 measuring points per meter and the measuring speed is $3 \sim 4 \mathrm{~km} / \mathrm{h}$. The measuring instrument is shown in Figure 1. The existing CAT corrugation measurement car adopts the measurement method of single rail, that is, only one rail can be measured at a time. Therefore, one track needs to be measured once back and forth. A section including uplink and downlink has to be measured twice. The field test is shown in Figure 2.

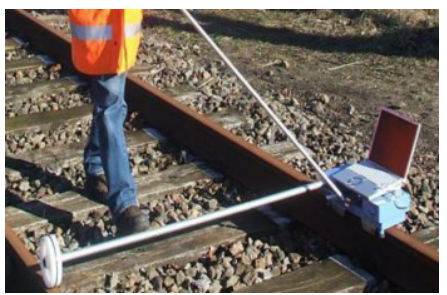

Figure 1. Rail corrugation measuring instrument.

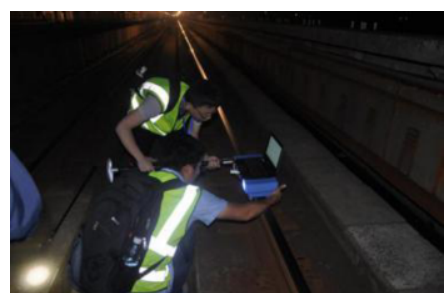

Figure 2. Field testing.

\section{Analysis on Amplitude Distribution Characteristics of Short Wave Irregularity on Rail Surface}

The amplitude distribution law of rail surface short wave irregularity will affect wheelrail interaction force, so it is necessary to study and analyze the amplitude distribution probability of rail surface short wave irregularity. Generally various special probability 
distribution functions, such as Poisson distribution, normal distribution and Bernoulli distribution, need to be introduced when studying the distribution characteristics of track irregularity amplitude. Normal distribution is widely used in engineering due to its centralization, symmetry and uniform variation [12]. By using of normal distribution the spatial distribution characteristics of short wave irregularity amplitude are analyzed in this paper.

\subsection{Distribution Characteristics of Rail Surface Irregularity Amplitude}

The amplitude data of rails in each section of Shanghai Rail Transit Line 11\# are statistically classified and the corresponding amplitude distribution law is analyzed. The results are shown in Table 1. From Table 1, it can be seen that the maximum absolute value of rail surface short wave irregularity in each section of Line 11 is distributed in the range of $1.0 \sim 2.5 \mathrm{~mm}$, and most of it is in the range of $2.0 \sim 2.5 \mathrm{~mm}$. This means that the distribution range of rail surface short wave irregularity amplitude is different in various sections of the same line. In the same section, there are also differences in the amplitude distribution between the uplink and downlink, and even the amplitudes of the irregularity produced by the two rails in the same direction are different.

Table 1. Amplitude distribution interval and probability statistics of rail surface irregularity

\begin{tabular}{|c|c|c|c|c|}
\hline Line & & & $\begin{array}{c}\text { Distribution interval } \\
\text { amplitude }\end{array}$ & $\begin{array}{c}\text { Distribution } \\
\text { probability (\%) }\end{array}$ \\
\hline \multirow{2}{*}{ Jiading Xincheng -Malu } & \multirow{2}{*}{ Downlink } & Rail 3 & {$[-2.5,2.5]$} & 99.9955 \\
\hline & & Rail 4 & {$[-2.5,2.5]$} & 99.9944 \\
\hline \multirow{4}{*}{ Malu-Nanxiang } & \multirow{2}{*}{ Uplink } & Rail 1 & {$[-2.0,2.0]$} & 99.9953 \\
\hline & & Rail 2 & {$[-2.0,2.0]$} & 99.9948 \\
\hline & \multirow{2}{*}{ Downlink } & Rail 3 & {$[-2.0,2.0]$} & 99.9959 \\
\hline & & Rail 4 & {$[-2.0,2.0]$} & 99.9965 \\
\hline \multirow{4}{*}{$\begin{array}{l}\text { Nanxiang-Taopu } \\
\text { saemaul undong }\end{array}$} & \multirow{2}{*}{ Uplink } & Rail 1 & {$[-2.5,2.5]$} & 99.9950 \\
\hline & & Rail 2 & {$[-2.5,2.5]$} & 99.9940 \\
\hline & \multirow{2}{*}{ Downlink } & Rail 3 & {$[-2.0,2.0]$} & 99.9962 \\
\hline & & Rail 4 & {$[-2.0,2.0]$} & 99.9953 \\
\hline \multirow{4}{*}{ Zhenru-FengqiaoRoad } & \multirow{2}{*}{ Uplink } & Rail 1 & {$[-1.0,1.0]$} & 99.9892 \\
\hline & & Rail 2 & {$[-1.5,1.5]$} & 99.9946 \\
\hline & \multirow{2}{*}{ Downlink } & Rail 3 & {$[-1.5,1.5]$} & 99.9949 \\
\hline & & Rail 4 & {$[-1.5,1.5]$} & 99.9927 \\
\hline \multirow{4}{*}{ Liziyuan-Shanghai West } & \multirow{2}{*}{ Uplink } & Rail 1 & {$[-2.0,2.0]$} & 99.9947 \\
\hline & & Rail 2 & {$[-2.0,2.0]$} & 99.9954 \\
\hline & \multirow{2}{*}{ Downlink } & Rail 3 & {$[-1.5,1.5]$} & 99.9943 \\
\hline & & Rail 4 & {$[-2.0,2.0]$} & 99.9942 \\
\hline
\end{tabular}




\subsection{Statistics of Amplitude Frequency of Uplink and Downlink Irregularities on Zhenru- Fengqiao Road}

In order to further study the distribution characteristics of rail surface short wave irregularity amplitude, in this paper the measured data from Zhenru-Fengqiao section were analyzed. Statistical analysis is carried out on the frequency of rail surface short wave irregularity amplitude range within the main distribution range to understand the distribution of rail surface short wave irregularity amplitude. The results are shown in Figure 3. Each of $0.05 \mathrm{~mm}$ is a unit interval in the figure.

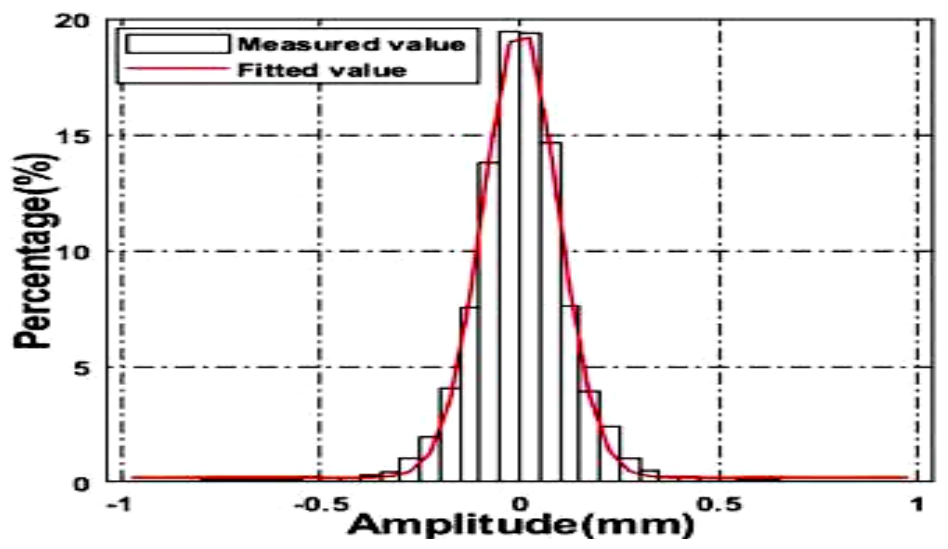

(a)Amplitude frequency statistics of uplink Rail 1

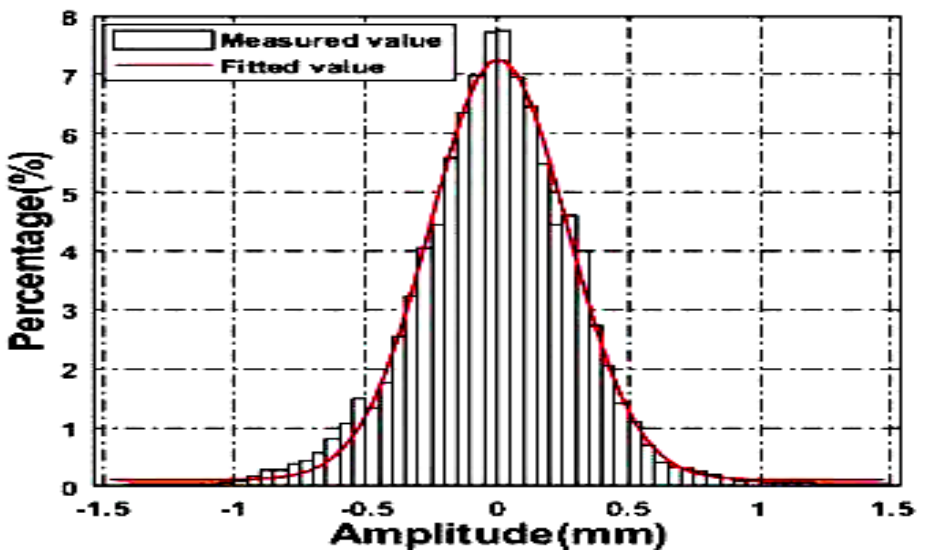

(b)Amplitude frequency statistics of uplink Rail 2 


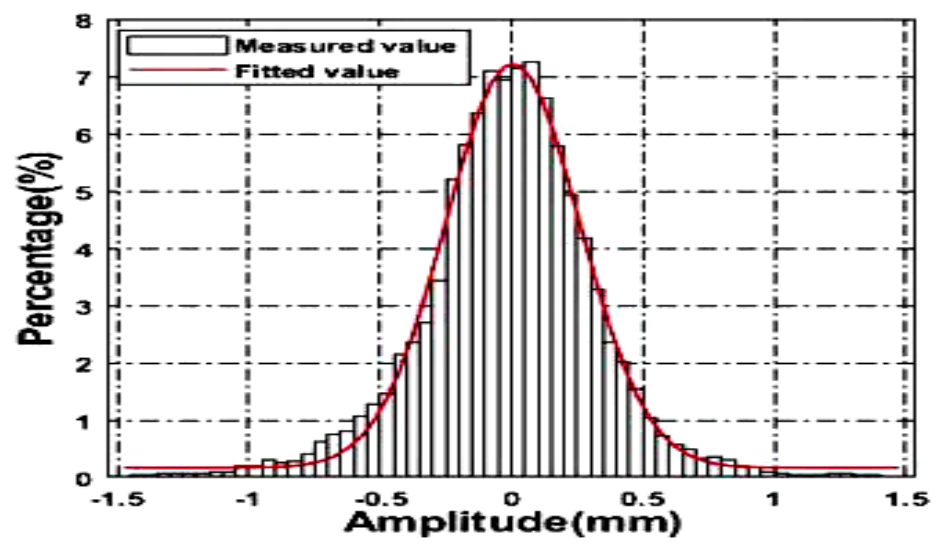

(c)Amplitude frequency statistics of downlink Rail 3

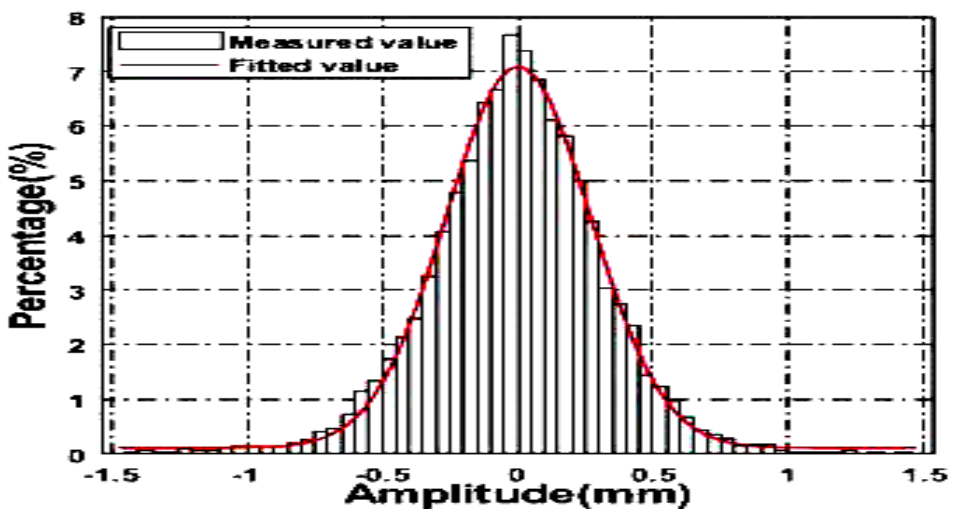

(d)Amplitude frequency statistics of downlink Rail 4

Figure 3. Statistics of amplitude and frequency of short wave irregularity on upper and lower rail surfaces on Zhenru-Fengqiao Road.

As can be seen from Figure 3, for the uplink rail 1, the main distribution range of amplitude is $[-1.0,1.0]$. The percentage of amplitude frequency in the distribution ranges of $[-0.05,0]$ and $[0,0.05]$ is the highest, which is $19.4129 \%$ and $19.3818 \%$ respectively. For the uplink rail 2 , the main distribution range of amplitude is $[-1.5,1.5]$. The percentage of amplitude frequency in $[-0.05,0]$ and $[0,0.05]$ ranges is the highest, which is $7.7318 \%$ and $7.7485 \%$ respectively. For the downlink rail 3 , the main distribution range of amplitude is $[-1.5,1.5]$. The percentage of amplitude frequency in the distribution ranges of $[0,0.05]$ and $[0.05,0.10]$ is the highest, which is $7.1520 \%$ and $7.2419 \%$ respectively. For the downlink rail 4, the main distribution range of amplitude is $[-1.5,1.5]$. The percentage of amplitude frequency in the distribution ranges of $[-0.05,0]$ and $[0,0.05]$ is the highest, which are $7.6609 \%$ and $7.3815 \%$ respectively. For the whole section, as the absolute value of the amplitude increases, the corresponding amplitude frequency decreases. The shape of the overall frequency distribution curve is high in the middle and low on both sides, with $\mathrm{x}=0$ as the axis of symmetry and symmetrical distribution on left and right, and the distribution characteristic is approximate to normal distribution. 


\section{Power Spectrum Estimation and Analysis of Short Wave Irregularity on Rail Surface}

The measured data used in this paper do not distinguish between the welded joint area and the non-joint area in measurement. Conventional power spectral density estimation methods may cause large errors in the calculation results of the joint area. Therefore, the maximum entropy spectrum method is used to estimate the power spectral density of each line section.

\subsection{Principle of Maximum Entropy Spectroscopy}

Maximum entropy spectroscopy is a method which extrapolates unknown autocorrelation function value by using known autocorrelation function value under the criterion of maximum entropy, eliminating the artificial assumption on unknown data, making the parts other than known autocorrelation function has the strongest randomness, thus making the result of spectrum estimation more reasonable [13].

According to the research results of Jin Xinyang [14], Ma Jingku [15], etc. and Ji Peirong [16], the formulas for calculating the power spectral density by the maximum entropy method are as follows:

$$
\widetilde{G}_{x}(f)=\frac{p_{m} \cdot \Delta t}{\left|1+\sum_{m=1}^{L} a_{m} e^{-j \cdot 2 \pi f m \Delta t}\right|^{2}}
$$

$a_{m}:$ predictive error filtering coefficient;

$p_{m}:$ white noise sequence;

$L$ : Filtering factor length.

By V.D.Bos verification [17], the maximum entropy spectrum estimation is equivalent to the estimation spectrum of the autoregressive model in the calculation process, i.e., the autoregressive model can be used to approximate the maximum entropy spectrum and obtain a more accurate solution. According to this idea, Qian Shaoxin[18] has completed the transformation between maximum entropy power spectrum and AR spectrum and put forward a new autoregressive model spectrum:

$$
S_{A R}(f)=\frac{2 \sigma^{2}}{\left|1+\sum_{m=1}^{M} a_{k} e^{-j \cdot 2 \pi f m \Delta t}\right|^{2}}
$$

$M$ : order of AR Model;

$\sigma^{2}$ : variance of white noise sequence;

$a_{k}:$ AR Model Coefficient.

In the new autoregressive model spectrum, the determination of AR model order is very important, which directly affects the change trend of power spectral density curve. If the order is too small, the power spectrum density curve will tend to be smooth, which results in some data can not be recognized. If the order is too large, the power spectrum density curve will oscillate and generate false peaks, which will also lead to a 
sharp increase in the amount of calculation. In this paper, the order of AR model is 0.01 times of the length of irregularity data signal according to empirical formula during the calculation of rail surface short wave irregularity spectrum.

\subsection{Analysis of Short Wave Irregularity Spectrum on Rail Surface of Liziyuan- Shanghai West Station}

In order to study and analyze the influence of track structure on the value of power spectrum density of rail surface short wave irregularity. This paper takes the section of Liziyuan-Shanghai West Station as an example, divides each rail into several sections according to the different track structures, solves the power spectrum density curve of each rail section separately, and compares it with the short wave irregularity power spectrum of Shi-Tai Line proposed by Wang Lan [19] and the roughness spectrum introduced by Japanese scholar Sato [20] when analyzing high-frequency vibration of wheel-rail system. The results are shown in Figure 4-5 (the power spectrum proposed by Wang Lan in the figure is named as Standard 1 and the Sato spectrum is named as Standard 2).

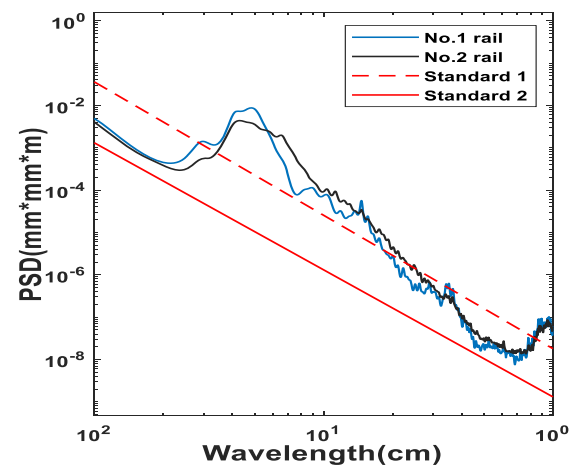

(a)Circular tunnel floating plate, DTIII-2 fasteners, conventional solid bed.

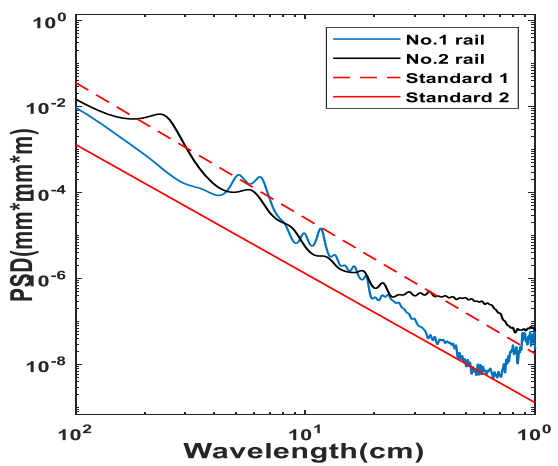

(b)Support block, DTIII-2 fasteners, conventional solid bed.

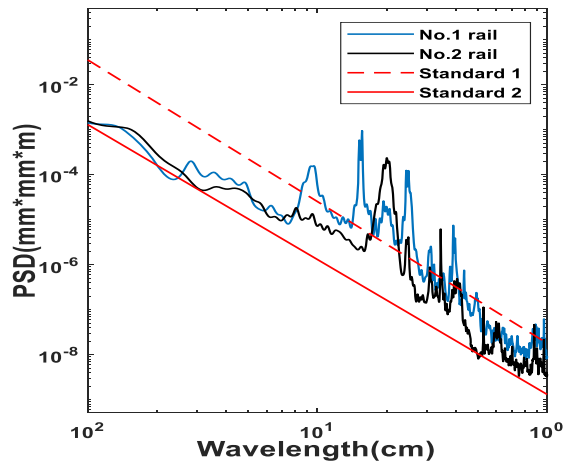

(c)Support block, track shock absorber fasteners, conventional solid bed.

Figure 4. Power spectrum curve of short wave irregularity on uplink track surface of Liziyuan-Shanghai west station. 
As can be seen from Figure 4(a), in the uplink section, the overall trend of the power spectral density curve are basically the same of the rail 1 and rail 2 with circular tunnel floating plate, DTIII-2 fasteners and conventional solid bed. In the whole wavelength range, the power spectrum density values of rail surface short wave irregularity of rail 1 and rail 2 are greater than Sato roughness spectrum. In the wavelength range of $2.5 \mathrm{~cm} \sim 9.0 \mathrm{~cm}$ and $80 \mathrm{~cm} \sim 100 \mathrm{~cm}$, rail 1 and rail 2 are lower than short wave spectrum of Wang Lan. There is a peak in the wavelength range of 50 $\mathrm{cm} \sim 60 \mathrm{~cm}$, which is higher than the short wave spectrum of Wang Lan. From Figure 4(b), it can be seen that the trend of power spectrum density curve of rail 1 and rail 2 with supporting blocks, DTIII-2 fasteners and conventional solid bed is basically the same in the wavelength range of $8.5 \mathrm{~cm} \sim 75 \mathrm{~cm}$, and between the Sato roughness spectrum and the short wave spectrum of Wang Lan. In the wavelength range of $1 \mathrm{~cm} \sim 2 \mathrm{~cm}$, the power spectrum density value of rail 1 is higher than that of Wang Lan. In the wavelength range of $1 \mathrm{~cm} \sim 7 \mathrm{~cm}$ and $80 \mathrm{~cm} \sim 92 \mathrm{~cm}$, the power spectrum density value of rail 2 is higher than that of Wang Lan. From Figure 4(c), it can be seen that the power spectrum density curves of rails 1 and 2 with support block, rail damper fasteners and conventional solid bed are higher than those of Wang Lan in the wavelength range of $8.3 \mathrm{~cm} \sim 9.6 \mathrm{~cm}$, while the others are basically between Sato roughness spectrum and Wang Lan short wave spectrum.

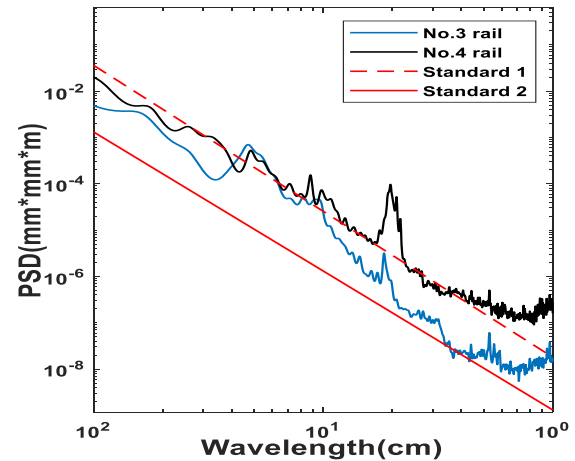

(a)Circular tunnel floating plate, DTIII-2 fasteners, conventional solid bed.

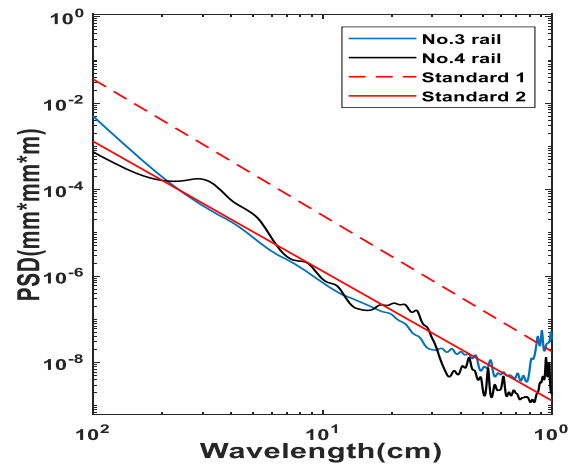

(b)Support block, DTIII-2fasteners, conventional solid bed.

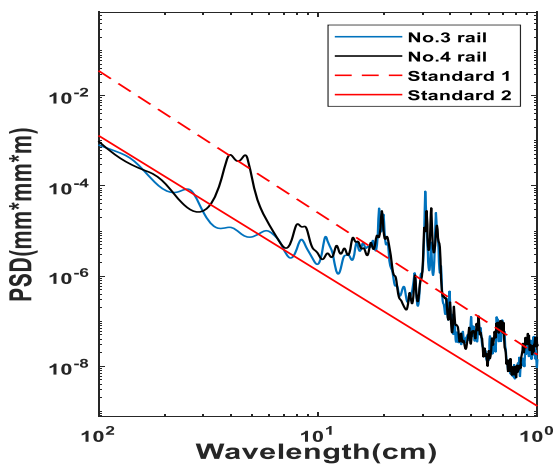

(c)Support block, LORD fasteners, conventional solid bed.

Figure 5. Power spectrum curve of short wave irregularity on downlink track surface of Liziyuan-Shanghai west station. 
As can be seen from Figure 5(a), in the downlink section, the overall trend of the power spectral density curves of rail 3 and rail 4 with circular tunnel floating plate, DTIII-2 fastener, conventional solid bed are basically the same. The power spectrum density value of rail 3 is basically between Sato roughness spectrum and Wang Lan short wave spectrum in the whole wavelength range, while rail 4 is basically similar to Wang Lan short wave spectrum in the wavelength range of $1 \mathrm{~cm} \sim 43 \mathrm{~cm}$. As can be seen from Figure 5(b), the power spectrum density values of rail 3 and rail 4 with support block, DTIII-2 fastener and conventional solid bed are similar to those of the Wang Lan in the whole wavelength range. As can be seen from Figure 5(c), the power spectrum density values of rail 3 and rail 4 with support block, LORD fasteners and conventional solid bed are lower than the Sato roughness spectrum in the wavelength range of $91 \mathrm{~cm} \sim 100 \mathrm{~cm}$. In the wavelength range of $42 \mathrm{~cm} \sim 79 \mathrm{~cm}$, there is an obvious wave peak of rail 4 compared with rail 3 and the peak value is higher than the short wave spectrum of Wang Lan. In the wavelength range of $1 \mathrm{~cm} \sim 7.2 \mathrm{~cm}$, the rail 3 and rail 4 are basically between the Sato roughness spectrum and the short wave spectrum of Wang Lan.

By comparing Figure 4 with Figure 5, the power spectrum density curves of rails with support block, DTIII-2 fastener and conventional solid bed are smoother than other track structures, and the state of track surface irregularity is better. The track structure of floating plate of circular tunnel, DTIII-2 fastener and conventional solid bed take the second place. The track structure of support block, track damper fastener, conventional solid bed and support block, LORD fastener and conventional solid bed is the worst. When the wavelength is small, the power spectrum density curve oscillates greatly for several times, which has obvious narrowband random spectrum characteristics.

\section{Conclusions}

Based on the analysis of the rail surface short wave irregularities data from the field test, some conclusions can be drawn:

(1)The maximum absolute value of short wave irregularity amplitude of rail surface in each section of Line 11 is distributed in the range of $1.0 \sim 2.5 \mathrm{~mm}$, and most of it is in the range of $2.0 \sim 2.5 \mathrm{~mm}$. The amplitude distribution of uplink and downlink rails and left and right rails of the same line will make a small difference.

(2)With the increase of absolute value of short wave irregularity on rail surface, the corresponding amplitude frequency decreases. The shape of the general frequency distribution curve is high in the middle and low on both sides. The distribution characteristic is similar to the normal distribution.

(3)For Liziyuan-Shanghai West Station section, because the maximum amplitude distribution of rail 3 is less than rail 1,2 and 4, the short wave irregularity power spectrum of rail surface is also smaller than that of the other 3 rails, which indicates that the research on the distribution characteristics of the irregularity amplitude has certain reference effect on power spectrum density.

(4)For Liziyuan-Shanghai West Station section, the irregularity of rail surface of supporting block type is better than that of floating plate of circular tunnel under the same type of fastener and ballast bed. The irregularity of rail surface of DTIII-2 fastener is better than that of rail damper fastener and LORD fastener under the same type of track and ballast bed. 


\section{Acknowledgements}

The authors wish to acknowledge the support and motivation provided by Project supported by National Natural Science Foundation, China (Grant No. 51668019), Natural Science Foundation of Jiangxi Province Science and Technology department (No.20171BAB206057) and Science Foundation of Jiangxi Province Education department (No.GJJ180297).

\section{References}

[1] Shanghai Railway Bureau, Tongji University. Research on Dynamic Analysis and Grinding Technology of short wave Irregular Rail of Speed-up Rail Line. Shanghai: Tongji University, 2009.

[2] Zhou Y. Analysis of rail surface roughness level spectrum for urban rail transit. Urban Mass Transit, 2014, 17(4):18-22.

[3] Nielsen JCO. Rail roughness level assessment based on high-frequency wheel-rail contact force measurements. Noise and Vibration Mitigation, 2008,99:355-362.

[4] Wei HL, Lian SL, Zhou Y. Experimental study on influence of rail surface irregularity on track structure vibration characteristics of high-rise support block. China Railway Science,2011,32(06):22-27.

[5] Chen MM, Lian SL, Cheng XP. Experimental research on the relationship between short wavelength irregularity of rail surface on jointer areas and P1, P2. Journal of Lanzhou Jiaotong University, 2011,30(01):59-63.

[6] Li ZW, Lei XY, Gao L. A new method for track short wave irregularity simulation. Journal of Traffic and Transportation Engineering,2016,16(01):37-45.

[7] Niu LB, Jin G, Liu JC, Zu HL. Application of the wheel-rail force in track short wave irregularity detection. Railway Engineering,2019,59 (08):133-139.

[8] EN ISO 3095. Railway application acoustics measurement of noise emitted by rail bound vehicles. 2005.

[9] Hiensch M, Nielsen JCO, Verheijen E. Rail corrugation in the Netherlands-measurements and simulations. Wear,2002,253(1-2):140.

[10] Verheijen E. A survey on roughness measurements. Journal of Sound Vibration,2006,293(3-5):784.

[11] Nielsen JCO. Rail roughness level assessment based on high-frequency wheel/rail contact force measurement//Noise and Vibration Mitigation for Rail Transportation Systems. Notes on Numerical Fluid Mechanics and Multidisciplinary Design. Berlin:Springer,2008:355-362.

[12] Fang KT, He P, Yang J. Representation point set of statistical distribution and its application. Scientia Sinica (Mathematica),2020,50(09):1149-1168.

[13] Tian ZY. Research and application of maximum entropy spectrum method for weak signal recognition in reflective earthquake. China University of Geosciences (Beijing),2019.

[14] Jin XY. Maximum entropy spectrum method and its application in random signal processing. Building Science, 1989(05):24-28.

[15] Ma JK, Li ZQ, Mu JH. Application of maximum entropy spectrum method in vibration signal analysis. Journal of Northeast Heavy Machinery Institute,1996(02):141-145.

[16] Ji PR, He ZY. Fast calculation of order and spectral value of maximum entropy spectral model. Journal of Wuhan University of Hydraulic and Electrical Engineering (Yichang),1997(01):30-34.

[17] Wu WS. Recursive least square algorithm for maximum entropy spectrum analysis. Journal of Shanghai Polytechnic University, 1987(02): 37-42.

[18] Qian SX, Liu WL, Yao FC. Application of maximum entropy principle in seismic data processing. Oil Geophysical Prospecting,1984(04):295-306.

[19] Wang L. Random vibration theory of track structures and its application in track structural damping. Doctoral Dissertation, China Academy of Railway Sciences, 1988.

[20] Sato Y. Study on high-frequency vibration in track operation with high-speed trains. Quarterly Reports, $1977,18(3): 22-27$. 